\title{
Examination of Aging in the Treatment of Abdomino- Pelvic Disorders Using the Lormand-Walsh Program
}

\author{
Marc Bonis*, Lormand J and Walsh C \\ University of New Orleans, USA
}

*Corresponding author: Marc Bonis, Human Performance and Health Promotion Program, ELCF Department ED 348K, University of New Orleans, USA

To Cite This Article: Marc Bonis, Lormand J, Walsh C. Examination of Aging in the Treatment of Abdomino-Pelvic Disorders Using the Lormand-Walsh Program. 2020 - 9(6). AJBSR.MS.ID.001444. DOI: 10.34297/AJBSR.2020.09.001444.

Received: 笽 June 15, 2020; Published: 制 August 04, 2020

\begin{abstract}
Purpose: Examine the effects of aging in the treatment of abdomino-pelvic disorders using the Lormand-Walsh Program (LWP).

Subjects: Forty-one women (Age $=43.2+9.5 \mathrm{yrs})$, twenty under 40 yrs old $(37.1+4.6 \mathrm{yrs})$ and twenty-one $40 \mathrm{yrs}$ and older $(49.1+9.4 \mathrm{yrs})$.

Methods: Subjects were assessed. The LWP was applied. Subjects were retested.

Results: IBM SPSS Version 24 Repeated Measures ANOVA analyses indicated significant statistical improvement and efficacy using the LWP in all 9 assessments. One-Way ANOVA analyses between the two age groups revealed no statistical significance except for the PFDI-20 bladder component test scores, $\mathrm{F}(1,40)=7.4, \mathrm{p}=0.01$; however, after the LWP intervention the two age groups of the PFDI-20 bladder component re-test scores no longer revealed statistically significant differences, $\mathrm{F}(1,40)=1.2, \mathrm{p}=0.29$.
\end{abstract}

Conclusions: Study indicated no deleterious effects due to aging using LWP.

Key words: Pelvic floor disorders; Low back pain; Pelvic floor muscles

\section{Introduction}

Low back pain (LBP) is a condition of localized pain to the lumbar spine whose etiology is commonly unknown [1]. Pelvic floor disorders (PFD) occur when the muscles that comprise the pelvic floor fail to properly contract. This can cause urinary incontinence, pelvic organ prolapse, fecal incontinence, or other sensory and emptying abnormalities of the lower urinary and GI tracts [2]. Current evidence shows that individuals with low back pain have a significant decrease in pelvic floor function compared to individuals without LBP [3]. Over $25 \%$ of all women and more than a third over the age of 65 experience PFD. Even though it is a physiological problem, the psychosocial impact of PFD can be much more detrimental to the patient's quality of life. Over the next 30 years chronic health problems associated with PFD are projected to increase by $50 \%$ due to the increasing numbers of women reaching age 65 [4]. PFD does not typically have one specific cause.
Pregnancy/childbirth, age, hormonal changes, obesity, lower UTI, and pelvic surgery are major risk factor [5]. Additionally, anatomical, physiological, genetic, reproductive and behavioral components can determine PFD developmental influences [1,4].

The pelvic floor forms the inferior border of the abdominopelvic cavity [4]. It supports the abdomino-pelvic organs. The pelvic floor muscles (PFM) function as a unit instead of individually contracting. They play an important role in maintaining and increasing intra-abdominal pressure during functional tasks such as lifting, sneezing, coughing, and laughing to prevent urinary and fecal incontinence $[3,6]$. Current evidence supports exercise protocols with the common goal of regaining neuromuscular control of the pelvic floor and deep abdominal muscles in a functional matter $[7,8]$. There is also strong evidence for PFM training as conservative treatment for stress urinary incontinence $[5,9]$. Treatment should 
also include education on healthy lifestyle habits to promote optimal functioning of the lumbopelvic stability system. Examples of these habits include good posture, maintenance of a healthy body weight, proper diet, routine exercise, and refraining from smoking [6].

The purpose of this study is to determine if age significantly impacts the improvements of symptoms from pelvic floor dysfunction and mild pelvic organ prolapse (Stage 1or 2) in women brought about by the specific exercise and behavioral interventions used in the Lormand-Walsh Program (LWP) [7].

\section{Methods}

The current research evaluated whether the age of the study participants affected the lumbo-pelvic improvements from the Lormand-Walsh Program, a protocol of behavioral modifications as well as a specialized exercise program designed to improve the pain levels and quality of life symptoms of pelvic floor dysfunction and mild pelvic organ prolapse in women [7]. These symptoms include low back pain, hip pain, pelvic pressure, pelvic pain with intimacy and/or the use of tampons, bladder and/or bowel leakage with laughing, coughing, sneezing, jumping, bladder and/ or bowel urgency and frequency. The Lormand-Walsh Program is an effective assessment/intervention program designed to avoid invasive techniques. The assessment component consists of three assessment questionnaires along with some hands-on noninvasive assessments. The intervention component consists of a combination of behavioral changes and a daily physical activity routine that takes approximately 15 -minutes to complete. The intervention component lasts 30-days.

\section{Participants}

The study consisted of 41 female subjects between the ages of 28-69 years old. The subjects were recruited as a convenience sample from the Greater New Orleans area through the use of informational fliers, Facebook video ads, email messages of the same flier, and community postings of the flier. Prior to participation in the study the participants signed a letter of informed consent stating that the study was voluntary and confidential and that all results would be kept in a locked, secure environment. No remuneration was provided to the participants. Initially 42 female subjects were recruited; however, during the course of the study one participant was dropped from the research due to non-compliance. For purposes of the study the participants were divided by age into two groups: Participants under 40 years old (20 participants) and those 40 years old and older ( 21 participants) The study was approved by the Institutional Review Board (IRB) of the University of New Orleans. See (Table 1) for the participants' physical characteristics.

Table 1: Physical Characteristics of Participants (mean \pm std dev).

\begin{tabular}{|c|c|c|c|c|c|}
\hline Group & N & Age (yrs) & Weight (kg) & Height (m) & BMI \\
\hline Total & 41 & $43.2 \pm 9.5$ & $70.5 \pm 14.4$ & $1.63 \pm 0.06$ & $26.3 \pm 5.0$ \\
\hline Grp 1 $(<40)$ & 20 & $37.1 \pm 4.6$ & $68.6 \pm 15.2$ & $1.64 \pm 0.07$ & $25.5 \pm 5.3$ \\
\hline Grp 2 $(>40)$ & 21 & $49.1 \pm 9.4$ & $72.4 \pm 13.7$ & $1.64 \pm 0.06$ & $27.0 \pm 4.6$ \\
\hline
\end{tabular}

\section{Participation Criteria}

Research inclusion consisted of women that exhibited symptoms of pelvic floor dysfunction or mild pelvic organ prolapse as defined by the assessment portion of the Lormand-Walsh Program. The assessment portion of the program consists of three measures, the Pelvic Floor Impact Questionnaire - Short Form 7 (PFIQ-7) [8,9], the Pelvic Floor Distress Inventory Questionnaire - Short Form 30 (PFDI-SF20) [9], and the Oswestry Low Back Pain (LBP) Disability Questionnaire [10], along with 3 physical assessments that include a pelvic alignment, a diastasis recti assessment, and a manual external pelvic floor muscle activation [11].

Both the PFIQ-7 and the PFDI-20 questionnaires have three components, bladder, bowel, and prolapse, each ranging in value from 0 (least impact) to 100 (most impact with a composite range of 0 to 300. The Oswestry Lower Back Pain Questionnaire ranges in value from 0 (least impact) to 100 (most impact).

Participants were excluded from the study if they were assessed to have greater than a 3 finger-width diastasis recti abdominal separation as measured by one of the co-investigators. Exclusion criteria ensured that those with advanced symptoms were referred for medical consultation. All participants had ample opportunity for questions to be answered as needed by the co-investigator clinicians during the live sessions.

\section{Procedure}

The participants were assessed to determine their level of pain and dysfunction to record the subjects' pre-intervention levels. The participants were then introduced to the behavioral and exercise portion of the Lormand-Walsh Program. They were instructed in specific behavioral modifications and were taught an exercise program over the course of eight live group sessions, 45 minutes each which included a 10-15 exercise intervention. One or both of the co-investigators, an exercise physiologist and a physical therapist, provided verbal instruction as well as demonstrations and tactile assistance as needed to ensure safe and correct performance of the behavioral interventions and exercises. Participants were also required to perform a home exercise program at least 3 additional days per week. In summary, the participants performed the exercise program 5 times, 2 in the clinic and 3 at home with 2 
days off each week. Participants performed the exercise program 20 times during the 30-day intervention period. Finally, the participants were re-assessed after the procedure was completed to determine if there were any changes in pain and/or dysfunction. The participants were also provided with on-demand access to videos of the co-investigator clinicians teaching / reviewing the exercises. See (Table 2) for the participants' mean test and retest assessment questionnaire scores.

\section{Limitations}

The 3 additional-day per week home exercise portion of the procedure was self-administered and self-reported by the study's participants.

\section{Analysis}

IBM SPSS Version 24 was used to analyze the study's data. The level of significance for the analyses was set at $p=0.05$. One-Way Repeated Measures ANOVA was applied to compare the mean test scores to the mean retest scores of the same participants for the three questionnaires' scores and the three component test scores of the PFIQ-7 and PFDI-20 questionnaires. The two-level assumption of sphere city was met in all of the repeated measures analyses. In all three questionnaires and all of the three questionnaire components from PFIQ-7 and PFDI-20 (a total of 9 different assessments) the mean retest scores were significantly improved from the mean test scores after the LWP intervention was conducted. See (Tables 2 and 3).

\begin{tabular}{|c|c|c|c|}
\hline Table 2: Assessment Questionnaire \& Component Mean Test and Re-Test Scores (mean \pm std dev). \\
\hline Questionnaire & N & Test & Re-Test \\
\hline PFIQ-7 & 41 & $40.2 \pm 36.3$ & $18.4 \pm 28.4$ \\
\hline 7- Bladder & 41 & $15.2 \pm 18.4$ & $5.8 \pm 12.1$ \\
\hline 7 - Bowel & 41 & $8.0 \pm 11.5$ & $4.6 \pm 11.0$ \\
\hline 7 - Prolapse & 41 & $17.1 \pm 22.2$ & $7.9 \pm 14.2$ \\
\hline PFDI-20 & 41 & $77.0 \pm 54.7$ & $13.5 \pm 14.8$ \\
\hline 20 - Bladder & 41 & $33.5 \pm 25.0$ & $11.7 \pm 14.7$ \\
\hline 20 - Bowel & 41 & $23.3 \pm 21.9$ & $10.6 \pm 14.3$ \\
\hline 20-Prolapse & 41 & $20.2 \pm 16.3$ & $9.7 \pm 7.8$ \\
\hline Oswestry & 41 & $15.7 \pm 12.4$ & \\
\hline
\end{tabular}

\begin{tabular}{|c|c|c|c|c|c|}
\hline Questionnaire & $\mathbf{N}$ & Mauchly's W & Analysis & Partial $\eta^{2}$ & Pwr \\
\hline PFIQ-7 & 41 & 1 & $\mathrm{~F}(1,40)=22.5, \rho=.000$ & 0.36 & 0.996 \\
\hline 7-Bladder & 41 & 1 & $\mathrm{~F}(1,40)=15.2, \rho=.000$ & 0.276 & 0.968 \\
\hline 7 - Bowel & 41 & 1 & $\mathrm{~F}(1,40)=6.29, \rho=.016$ & 0.136 & 0.687 \\
\hline 7 - Prolapse & 41 & 1 & $\mathrm{~F}(1,40)=12.0, \rho=.001$ & 0.231 & 0.923 \\
\hline PFDI-20 & 41 & 1 & $\mathrm{~F}(1,40)=22.5, \rho=.000$ & 0.36 & 0.996 \\
\hline 20 - Bladder & 41 & 1 & $\mathrm{~F}(1,40)=37.6, \rho=.000$ & 0.491 & 1 \\
\hline 20 - Bowel & 41 & 1 & $\mathrm{~F}(1,40)=21.9, \rho=.000$ & 0.354 & 0.995 \\
\hline 20 - Prolapse & 41 & 1 & $F(1,40)=27.0, \rho=.000$ & 0.403 & 0.999 \\
\hline Oswestry & 41 & 1 & $F(1,40)=21.4, \rho=.000$ & 0.349 & 0.995 \\
\hline
\end{tabular}

One-Way ANOVA was used to compare the age subgroups of the mean test scores, and to also compare the subgroups of the mean retest scores. In all three questionnaires and all of the questionnaire components from PFIQ-7 and PFDI-20 the "under-40" mean subgroup \#1 test scores were better scores than the "over-40" mean subgroup \#2 test scores; however, they were not significantly different from each other, except for the PFDI-20 Bladder Component mean score. In that case the mean test scores of the "under-40" subgroup \#1 had statistically significant better scores than the "over-40" subgroup \#2. Levene's Homogeneity assumption was met for this analysis. See (Table 4). However, after the LWP application the mean retest scores of the "under-40" subgroup \#1 no longer had statistically significant better mean retest scores than the "over-40" subgroup \#2, even though the mean retest scores of the younger subgroup was better than the mean retest scores of the older subgroup. Levene's Homogeneity assumption was also met for this analysis. See (Table 5).

\begin{tabular}{|c|c|c|}
\hline \multicolumn{3}{|c|}{ Table 4: One-Way ANOVA PFDI-20 Bladder Component. } \\
\hline \multicolumn{3}{|c|}{ Sub-Group Comparison of Mean Test Scores } \\
\hline Description & $\mathrm{N}$ & Mean \pm SD Test Scores \\
\hline Group 1 & 20 & $25.2 \pm 16.9$ \\
\hline Group 2 & 21 & $41.0 \pm 20.2$ \\
\hline Total & 41 & $33.3 \pm 20.1$ \\
\hline Statistical Analysis & \multicolumn{2}{|c|}{$\mathrm{F}(1,40)=7.4, \rho=0.01$} \\
\hline
\end{tabular}




\begin{tabular}{|c|c|c|}
\hline \multicolumn{3}{|c|}{ Table 5: One-Way ANOVA PFDI-20 Bladder Component. } \\
\hline \multicolumn{3}{|c|}{ Sub-Group Comparison of Mean Re-Test Scores } \\
\hline Description & $\mathrm{N}$ & Mean \pm SD Re-Test Scores \\
\hline Group 1 (<40yrs) & 20 & $10.9 \pm 13.4$ \\
\hline Group 2(>40 yrs) & 21 & $15.9 \pm 15.8$ \\
\hline Total & 41 & $13.5 \pm 14.8$ \\
\hline Statistical Analysis & \multicolumn{2}{|c|}{$\mathrm{F}(1,40)=1.2, \rho=0.29$} \\
\hline
\end{tabular}

\section{Discussion}

Although the younger participants in the study generally scored better than the older participants, the application of the LWP did not show any deleterious results as compared to age.

Statistical significance does not equate to clinical experience; however, the implications of these results are far-reaching, as the size of the population suffering with these symptoms includes at least $1 / 3$ of all women. The women who participated in the current study included women who had recently become mothers, those whose youngest children were teenagers, as well as a few women who had no children but had either suffered a back injury or were diagnosed with a lax connective tissue disorder (Ehlers-Danlos Syndrome). All of them benefited with statistically significant symptom relief.

The LWP is effective because it includes strengthening the foundational muscles of the body - the core. From a motor learning level, it re-activates deep support muscles of the core that have often lain dormant since a precipitating incident, including most often pregnancy and childbirth, but also a low back injury, or due simply to disuse over many years possibly as a result of a seated occupation or sedentary lifestyle. This program functionally and subconsciously re-activates the deep abdominal and pelvic floor muscles to work in a coordinated and synergistic fashion, which is the way they must fire in order to be effective.

Until now there has not been a highly effective option to reactivate the necessary core muscles to relieve the symptoms. The LWP addresses those concerns as a program that (once trained) can be performed independently to successfully significantly improve all of the symptoms addressed. It is cost- and time-effective and non-invasive, as well as pain-free, all appealing qualities to patients who are desperately searching for answers and relief from these debilitating symptoms. This procedure takes 10-15 minutes per day. These qualities stand in contrast to often recommended internal vaginal treatments or other more invasive or costly therapies.

\section{Recommendations}

Additional research is recommended using larger populations and more diverse age ranges to better critique advantages and the benefits of the Lormand-Walsh Program.

\section{Reference}

1. Eliasson K, Elfving B, Nordgren B, Mattsson E (2008) Urinary incontinence in women with low back pain. Man Ther 13(3): 206-212.

2. Nygaard I, Barber M, Brody DJ, Burgio KL, Kenton K, et al. (2008) Prevalence of symptomatic pelvic floor disorders in US women. JAMA 300(11): 1311-1316.

3. Arab A, Behbahani R, Lorestani L, Azari A (2010) Assessment of pelvic floor muscle function in women with and without low back pain using transabdominal ultrasound. Man Ther 15(3): 235-239.

4. Davis K (2010) Pelvic floor dysfunction: causes and assessment. Practice Nursing 21(7): 340-346.

5. Davis K, Kumar D (2003) Pelvic floor dysfunction: a conceptual framework for collaborative Patient-centred care. J Adv Nurs 43(6): 555-568.

6. Mohseni Bandpei M, Rahmani N, Behtash H, Karimloo M (2011) The effect of pelvic floor muscle exercise on women with chronic nonspecific low back pain. J Bodyw Mov Ther 15(1): 75-81.

7. Bonis M, Lormand J, Walsh C (2020) Immediate Effects of Exercise and Behavioral Interventions for Pelvic Floor Dysfunction and Lumbopelvic Pain. Journal of Womens Health Physical Therapy 44(2): 54-62.

8. Bonis M, Lormand J, Walsh C (2019) Using the Pelvic Floor Impact Questionnaire and lifestyle modifications to improve symptoms of pelvic floor dysfunction. American Journal of Biomedical Science \& Research 3(1): 23-27.

9. Barber M, Chen Z, Lukacz E, Markland A, Wai C, et al. (2011) Further Validation of the Short Form Versions of the Pelvic Floor Distress Inventory (PFDI) and Pelvic Floor Impact Questionnaire (PFIQ). Neurourol Urodyn 30(4): 541-546.

10. Fairbank J, Pynsent P (2000) The Oswestry Disability Index. Spine 25(22): 2940-2952.

11. Grewar H, McLean L (2008) The integrated continence system: a manual therapy approach to the treatment of stress urinary incontinence. Man Ther 13(5): 375-386. 